\title{
Relationship of Soluble Interleukin-6 Receptors With Asthma: A Mendelian Randomization Study
}

\begin{abstract}
Yoshihiko Raita ${ }^{1 *}$, Zhaozhong Zhu ${ }^{1}$, Carlos A. Camargo Jr. ${ }^{1}$, Robert J. Freishtat ${ }^{2,3,4}$, Debby $\mathrm{Ngo}^{5}$, Liming Liang $^{6,7}$ and Kohei Hasegawa ${ }^{1}$

${ }^{1}$ Department of Emergency Medicine, Massachusetts General Hospital and Harvard Medical School, Boston, MA, United States, ${ }^{2}$ Division of Emergency Medicine, Children's National Hospital, Washington, DC, United States, ${ }^{3}$ Department of Pediatrics, George Washington University School of Medicine and Health Sciences, Washington, DC, United States, ${ }^{4}$ Department of Genomics and Precision Medicine, George Washington University School of Medicine and Health Sciences, Washington, DC, United States, ${ }^{5}$ Pulmonary, Critical Care and Sleep Medicine, Beth Israel Deaconess Medical Center, Boston, MA, United States, ${ }^{6}$ Program in Genetic Epidemiology and Statistical Genetics, Department of Epidemiology, Harvard T.H. Chan School of Public Health, Boston, MA, United States, ${ }^{7}$ Department of Biostatistics, Harvard T.H. Chan School of Public Health, Boston, MA, United States
\end{abstract}

Purpose: Emerging evidence suggests a potential role of interleukin-6 pathways - trans-signaling with soluble interleukin-6 receptors-in the asthma

OPEN ACCESS

Edited by:

Mahmood Yaseen Hachim, Mohammed Bin Rashid University of Medicine and Health Sciences, United Arab Emirates

Reviewed by: Cynthia Koziol-White,

Rutgers, The State University of New Jersey, United States Khuloud Bajbouj, University of Sharjah, United Arab Emirates

*Correspondence: Yoshihiko Raita yraita1@mgh.harvard.edu

Specialty section: This article was submitted to

Pulmonary Medicine,

a section of the journal

Frontiers in Medicine

Received: 07 February 2021 Accepted: 17 March 2021

Published: 12 April 2021

Citation:

Raita Y, Zhu Z, Camargo CA Jr, Freishtat RJ, Ngo D, Liang $L$ and Hasegawa K (2021) Relationship of Soluble Interleukin-6 Receptors With Asthma: A Mendelian Randomization

Study. Front. Med. 8:665057. doi: 10.3389/fmed.2021.665057 pathobiology. Despite the evidence for their associations with asthma, the causal role of soluble interleukin- 6 receptors remains uncertain. We investigated the relations of soluble interleukin-6 receptors with asthma and its major phenotypes.

Methods: We conducted a two-sample Mendelian randomization study. As genetic instruments, we selected 33 independent cis-acting variants strongly associated with the level of plasma soluble interleukin-6 receptor in the INTERVAL study. To investigate the association of variants with asthma and its phenotypes, we used genome-wide association study data from the UK Biobank. We combined variant-specific causal estimates by the inverse-variance weighted method for each outcome.

Results: Genetically-instrumented soluble interleukin-6 receptor level was associated with a significantly higher risk of overall asthma (OR per one standard deviation increment in inverse-rank normalized soluble interleukin-6 receptor level, 1.02; 95\% Cl, 1.01-1.03; $P=0.004)$. Sensitivity analyses demonstrated consistent results and indicated no directional pleiotropy-e.g., MR-Egger $(\mathrm{OR}, 1.03 ; 95 \% \mathrm{Cl}, 1.01-1.05 ; P=0.002$; $P_{\text {intercept }}=0.37$ ). In the stratified analysis, the significant association persisted across asthma phenotypes - e.g., childhood asthma (OR, 1.05; 95\%Cl, 1.02-1.08; $P<0.001)$ and obese asthma (OR, 1.02; 95\% Cl 1.01-1.03; $P=0.007)$. Sensitivity analysis using 16 variants selected with different thresholds also demonstrated significant associations with overall asthma and its phenotypes.

Conclusion: Genetically-instrumented soluble interleukin-6 receptor level was causally associated with modestly but significantly higher risks of asthma and its phenotypes. Our observations support further investigations into identifying specific endotypes in which interleukin-6 pathways may play major roles.

Keywords: interleukin-6, soluble interleukin-6 receptor, trans-signaling pathway, asthma, Mendelian randomization, GWAS, UK Biobank 


\section{INTRODUCTION}

Among many immune components involved in the pathobiology of asthma, recent research has suggested a potential role of interleukin-6 (IL-6) signaling-the classic and trans-signaling pathways (1). The trans-signaling pathway starts with coupling with IL-6 and soluble IL-6 receptor (sIL-6R), and formation of a complex with the ubiquitously expressed membranebound glycoprotein 130, thereby activating downstream proinflammatory cascades-e.g., Janus kinase-signal transducer and activator of transcription (JAK-STAT) pathway (1). This IL6 trans-signaling pathway plays major roles in a range of inflammatory conditions (e.g., rheumatic diseases, inflammatory bowel diseases, obesity), and is the target of anti-IL-6 therapies (e.g., tocilizumab) (1).

Epidemiologic studies have found associations of an increased IL-6 level in the serum, sputum, and bronchoalveolar lavage fluid with asthma prevalence and its severity (2-4). Genomewide association studies (GWAS) have also reported that the single nucleotide polymorphism (SNP) rs2228145 (Asp ${ }^{358} \mathrm{Ala}$ ) a variant in $I L 6 R$ that increases IL-6R shedding and promotes IL-6 trans-signaling-is associated with asthma prevalence, asthma severity, and lower pulmonary function $(5,6)$. Moreover, rs4129267-which has a perfect linkage disequilibrium with rs2228145 above in European subjects-is also known as an asthma susceptibility locus (7). Despite the evidence on these associations which may suffer from unmeasured confounding, the causal role of sIL-6R in asthma (and hence the potential role of anti-IL-6R therapies) remains uncertain. To address the knowledge gap in the literature, we performed an instrumental variable analysis with genetic instruments (i.e., Mendelian randomization) to examine the effect of sIL-6R on asthma and its major phenotypes.

\section{METHODS}

This is a two-sample Mendelian randomization study using GWAS summary statistics from two large cohort studiesthe INTERVAL study (8) and UK Biobank (9-12). Detailed Methods can be found in the Supplementary Material. In brief, Mendelian randomization can provide unbiased causal estimates in an observation study because the genetic polymorphisms associated with the exposure (sIL-6R levels) are allocated randomly at conception and its causal inference is less susceptible to confounding and reverse causation (13).

\section{DATA SUMMARY}

\section{The Interval Study}

The INTERVAL study is a prospective cohort study that recruited approximately 50,000 blood donors aged $\geq 18$ years. For the proteomic profiling, randomly selected two non-overlapping sub-cohorts of 2,731 and 831 participants of European ancestry were enrolled. The levels of 2,994 plasma proteins were measured by the use of SomaLogic assays. A genome-wide protein quantitative trait loci (pQTL) analysis of 2,994 plasma proteins in 3,301 healthy adults of European ancestry was conducted
(8). Overall, 1,927 significant $\left(\mathrm{P}_{\mathrm{GWAS}}<1.5 \times 10^{-11}\right)$ associations between 764 genomic regions and 1,478 proteins were identified. The summary statistics data are publicly available at http://www. phpc.cam.ac.uk/ceu/proteins/.

\section{The UK Biobank}

The UK Biobank is a prospective cohort study that enrolled approximately 500,000 adults (aged 40-69 years at enrollment in 2006-2010), and collected comprehensive phenotypic data and performed genome-wide genotyping (9). The current analysis restricted the sample to 394,256 subjects of European ancestry to minimize population stratification (46,799 cases with asthma and 347,457 controls). In the current study, the primary outcome was (overall) asthma $(n=46,799)$. The secondary outcomes were six major asthma phenotypes: (1) childhood asthma (defined as age of onset $\leq 12$ years; $n=9,676)$ (12), (2) adult-onset asthma (defined as age of onset $\geq 26$ years; $n$ $=22,294$ ) (12), (3) allergic asthma (defined as asthma with an allergic disease-eczema, food allergy, and/or allergy rhinitis [identified by data fields 6152, 20002, 41202, 41204]; $n=$ $23,183)(10,11),(4)$ non-allergic asthma (defined as asthma without any allergic disease; $n=23,616$ ), (5) obese asthma (defined as BMI of $\geq 30 \mathrm{~kg} / \mathrm{m}^{2} ; n=13,550$ ), and (6) nonobese asthma (defined as BMI of $<30 \mathrm{~kg} / \mathrm{m}^{2} ; n=33,095$ ). We also identified shared controls $(n=347,457)$ with highquality genotyping and complete phenotype and covariate data for GWAS association analysis. All participants from this study provided UK Biobank-acquired informed consent and provided data according to the UK Biobank protocol. The institutional review board of Harvard University and Massachusetts General Hospital approved the study.

\section{Statistical Analysis}

As the genetic instruments, we identified 33 independent cis-acting variants strongly associated with plasma sIL-6R levels ( $\mathrm{P}_{\mathrm{GWAS}}<5 \times 10^{-6}, r^{2}<0.1,250 \mathrm{~kb}$ from IL6R; Supplementary Table 1) in the INTERVAL study (mean age, 44 years; female, $49 \%$ ) (8). All variants had an F-statistic of $>10$, without a significant association with major confounders (such as education status, smoking, and physical activity; Supplementary Table 1) nor a known pleiotropy in Ensembl, GWAS catalog, and PhenoScanner. Separately, using the UK Biobank data, we computed the GWAS statistics for asthma and six major asthma phenotypes, as previously described (10-12).

To investigate the association of variants with outcomes, we used GWAS summary statistics of the UK Biobank. We weighted the magnitude of association of each variant with outcomes by that with sIL-6R, and combined causal estimates of sIL$6 \mathrm{R}$ on each outcome by the inverse-variance weighted metaanalysis method with a random-effects model $(14,15)$ by using MendelianRandomization package (16).

In the sensitivity analyses, we first applied MR-Egger regression (17), MR Pleiotropy RESidual Sum and Outlier (MRPRESSO) test (18), and MR weighted median method (19). MREgger regression detects pleiotropy based on the assumption that the pleiotropic associations are independent from the genetic associations with the exposure (i.e., the instrument strength 
independent of direct effect [InSIDE] assumption) and provides corrected estimates. MR-PRESSO test (18) detects violation of the restriction exclusion criterion assumption and provides corrected estimates by removing variants which exhibit pleiotropy. MR weighted median method provides consistent estimates even when $50 \%$ of the information comes from invalid variants. We conducted MR-Egger regression and weighted median method using MendelianRandomization package (16) and MR-PRESSO using MRPRESSO package (18). Second, we also used morestringent $\mathrm{P}_{\mathrm{GWAS}}\left(\mathrm{P}_{\mathrm{GWAS}}<5 \times 10^{-8}\right)$ and linkage disequilibrium $\left(\begin{array}{ll}r^{2} & <0.02\end{array}\right)$ thresholds to select genetic instruments in order to examine the robustness of the inferences. We analyzed the data using $\mathrm{R}$ version 3.6.3 ( $\mathrm{R}$ foundation for Statistical Computing, Vienna, Austria).

\section{RESULTS}

Higher genetically-instrumented sIL-6R levels were associated with a modestly but significantly increased risk of overall asthma (OR per one standard deviation increment in inverserank normalized sIL-6R level, $1.02 ; 95 \% \mathrm{CI}, 1.01-1.03 ; P=$ 0.004 ; Figure 1) with the use of inverse-variance weighted metaanalysis method. Of the 33 genetic instruments, rs4129267an asthma susceptibility locus ${ }^{7}$ with a linkage disequilibrium of $r^{2}=1$ with $\mathrm{rs} 2228145$ (Asp ${ }^{358} \mathrm{Ala}$ ) (6) - had the largest weight on the Mendelian randomization estimate. The sensitivity analysis (Table 1) not only demonstrated consistent resultsMR-Egger (OR, 1.03; 95\%CI, 1.01-1.05; $P=0.002$ ), MRPRESSO $_{\text {corrected }}(\mathrm{OR}, 1.03 ; 95 \% \mathrm{CI}, 1.02-1.04 ; P<0.001)$, and MR weighted median (OR, 1.03; 95\%CI, 1.02-1.04; $P<0.001)$, but also indicated no directional pleiotropy in MR-Egger test $\left(P_{\text {intercept }}=0.37\right)$. Although the sensitivity analyses using MRPRESSO suggested potential pleiotropy $\left(P_{\text {global }}=0.02\right)$, the corrected MR-PRESSO yielded an estimate that is consistent with the primary analysis (OR, 1.03; 95\%CI, 1.02-1.04; $P<0.001)$ after removing variants with potential pleiotropy.

In the stratified analysis, the significant association persisted across the asthma phenotypes (Figure 2)-e.g., childhood asthma (OR, 1.05; 95\%CI, 1.02-1.08; $P<0.001)$ and obese asthma (OR, 1.02; 95\%CI, 1.01-1.03; $P=0.007$ ). Likewise, the sensitivity analysis also demonstrated consistent results (Table 1)-e.g., MR-Egger for childhood asthma (OR, 1.04; 95\%CI, 1.00-1.08; $P=0.04)$, MR-PRESSO for obese asthma (OR, 1.02; 95\%CI, 1.00-1.03; $P=0.001)$, and MR weighted median for childhood asthma (OR, 1.04; 95\%CI, 1.02-1.07; $P<0.001$ ). Although the sensitivity analyses using MR-PRESSO suggested potential pleiotropy for allergic asthma $\left(P_{\text {global }}=0.02\right)$ and non-obese asthma $\left(P_{\text {global }}=0.004\right)$, the corrected MR-PRESSO yielded an estimate that is consistent with the primary analysisallergic asthma (OR, 1.03; 95\%CI, 1.02-1.05; $P<0.001)$ and nonobese asthma (OR, 1.03; 95\%CI, 1.02-1.04; $P<0.001)$-after removing variants with potential pleiotropy.

Lastly, the sensitivity analysis using 16 variants selected by the use of more stringent thresholds $-\mathrm{P}_{\text {GWAS }}<5 \times 10^{-8}$ and linkage disequilibrium $\left(r^{2}<0.02\right)$-also demonstrated significant associations with overall asthma (OR, 1.03; 95\%CI, 1.02-1.04; $P<0.001$; Supplementary Figure 1) and its phenotypes-e.g., childhood asthma (OR, 1.05; 1.01-1.09; $P=0.01)$ and obese asthma (OR, 1.03; 95\%CI, 1.01-1.05; $P=0.01$; Figure 3).

\section{DISCUSSION}

In this two-sample Mendelian randomization study, we demonstrated that higher genetically-instrumented sIL-6R levels were associated with a modestly but significantly increased risk of overall asthma. The sensitivity analyses also showed consistent results. Our results are in line with recent findings that higher circulating IL-6 levels are associated with a greater

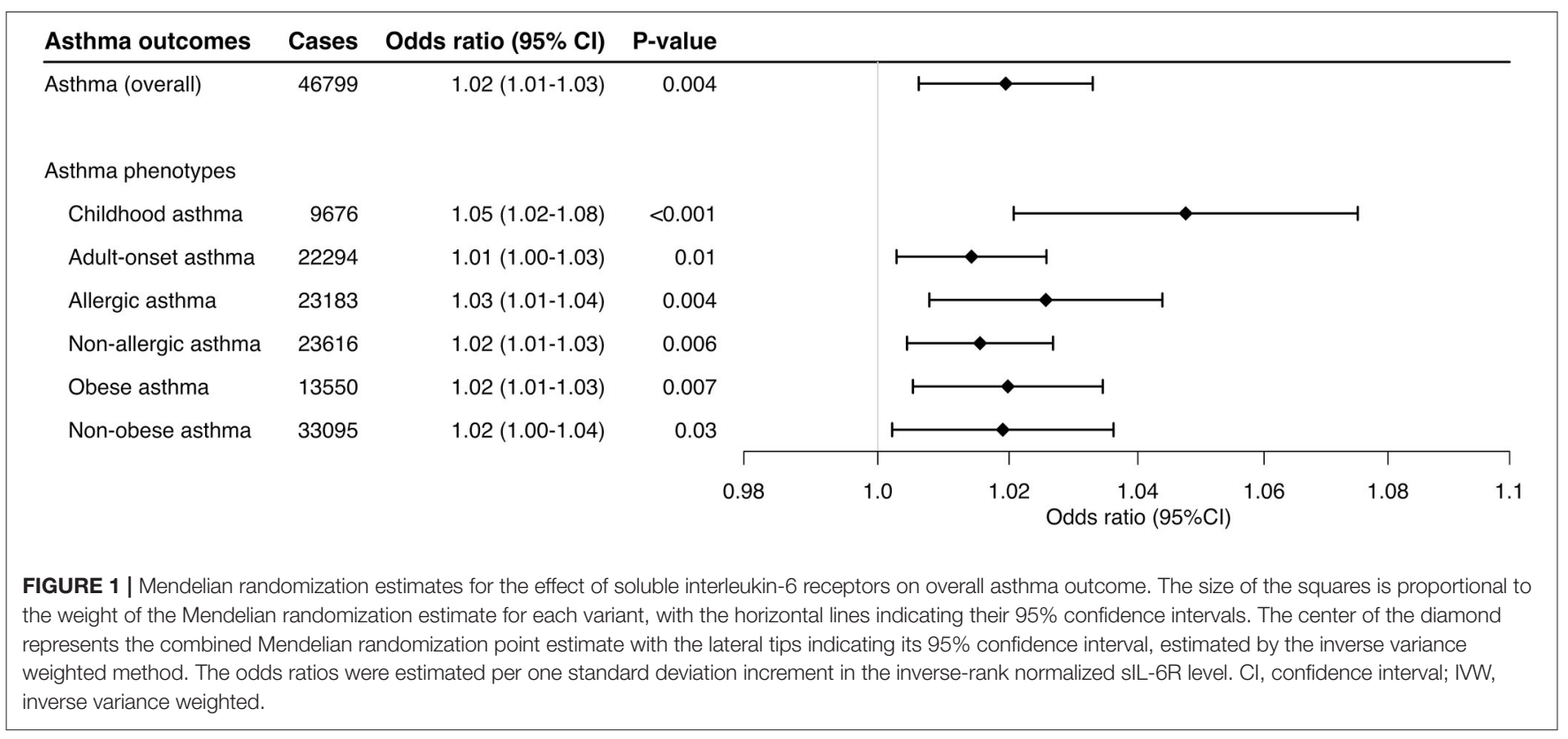




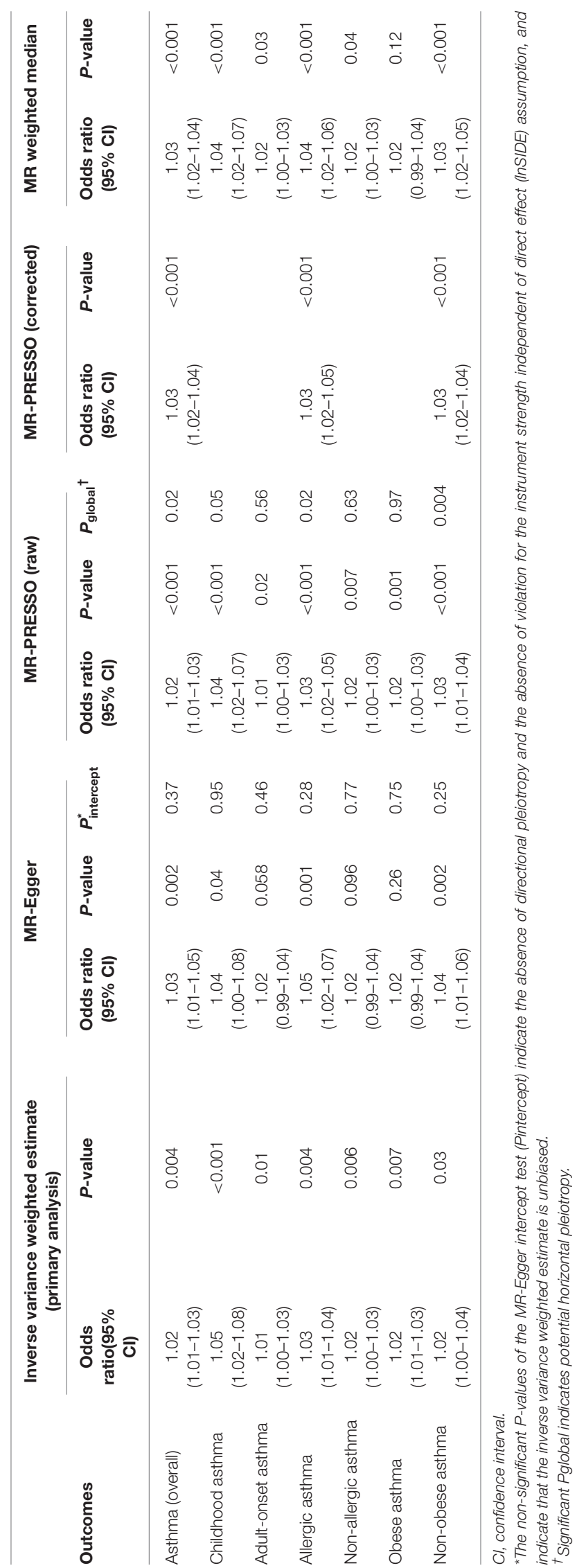

exacerbation risk both in children (20) and adults (21, 22). Additionally, genetic studies showed the associations of IL6Rrelated polymorphisms with asthma prevalence and severity, and lower pulmonary function $(5,6)$. For example, the Severe Asthma Research Program (SARP) cohorts found that rs2228145 is associated with a higher serum sIL-6R level, greater asthma severity, and lower pulmonary function in patients with severe asthma (6). However, their subsequent study also reported discordant results-e.g., no association of sIL-6R level with severity or exacerbation risk (22). The apparent discrepancies between these reports may be attributable to the differences in study design, setting, sample, analytical assumptions, or any combination of these factors. Regardless, the validity of the current study is buttressed by the use of the Mendelian randomization design. This design can mitigate unmeasured confounding and reverse causation that occur with conventional observational studies (13). The current analysis meets the assumptions of Mendelian randomization design in that we identified the genetic variants that are strongly associated with the sIL-6R level (the relevance assumption) and do not share common causes with asthma (the independence assumption), and in that we ensured no effects of known or unknown pleiotropy (the exclusion restriction assumption) (23). The current analysis using the data of two large cohorts corroborates the earlier reports, and extends them by investigating potential causal effects of sIL-6R on asthma and its phenotypes.

The mechanisms underlying our findings remain to be elucidated. For example, inflammatory signals (e.g., C-reactive protein, chemokine ligand 1 , IL- $1 \beta$, IL-8, tumor necrosis factor, bacterial lipopolysaccharides) promote alternative mRNA splicing and shedding of IL-6R from the cell surface, thereby producing sIL-6R and activating the IL-6 trans-signaling pathway (24-29). An analysis of U-BIOPRED data reported that adult patients characterized by IL- 6 trans-signaling-related epithelial gene signature had upregulated innate immune pathways, type 2 inflammation-independent eosinophilia, increased submucosal inflammation and airway remodeling, and higher asthma exacerbation rate (25). In patients with obese asthma, their airway inflammation is characterized by dominance of neutrophils and macrophages-major sources of sIL-6R in both lungs (5) and adipose tissue (30). Experimental asthma models also demonstrated that IL-6R inhibitors attenuate the airway inflammatory response characterized by mixed granulocytic infiltration with elevated IL-6 and IL-6R levels (31). These data collectively present a rationale for targeting the IL-6 trans-signaling pathway in asthma. We acknowledge that, in the current analysis, the observed magnitude of estimates was nominally small. Nevertheless, our findings encourage further investigations into identifying patients with specific asthma endotype(s) in which IL-6 pathways play major roles [e.g., patients with adiposopathy-"sick fat" (30)].

This study has potential limitations. First, misclassification of asthma and its phenotypes is possible, while it is unrelated to the measured sIL-6R levels in the current two-sample design. Therefore, this independent non-differential misclassification would have biased the inferences toward the null. Second, the 


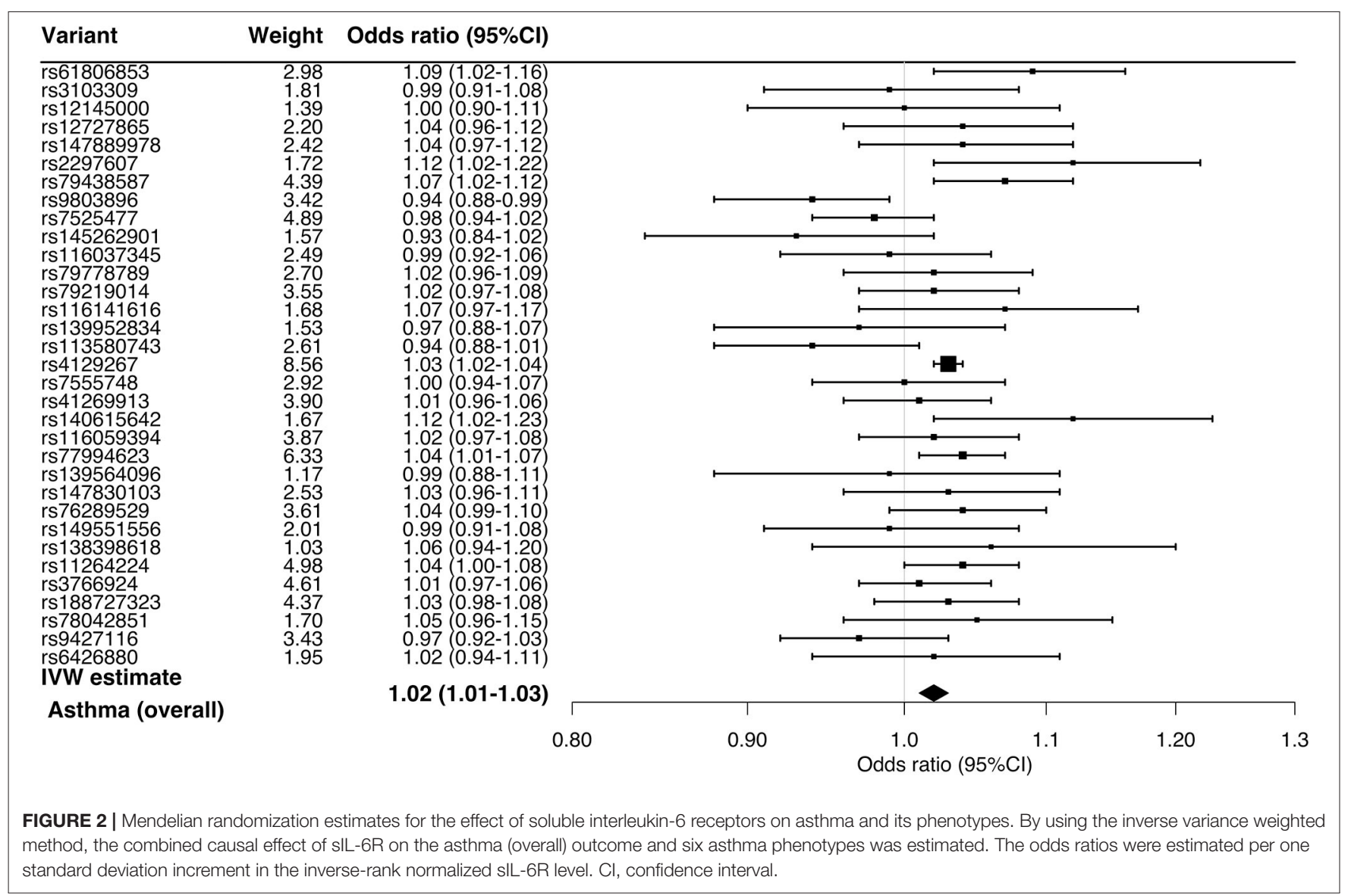

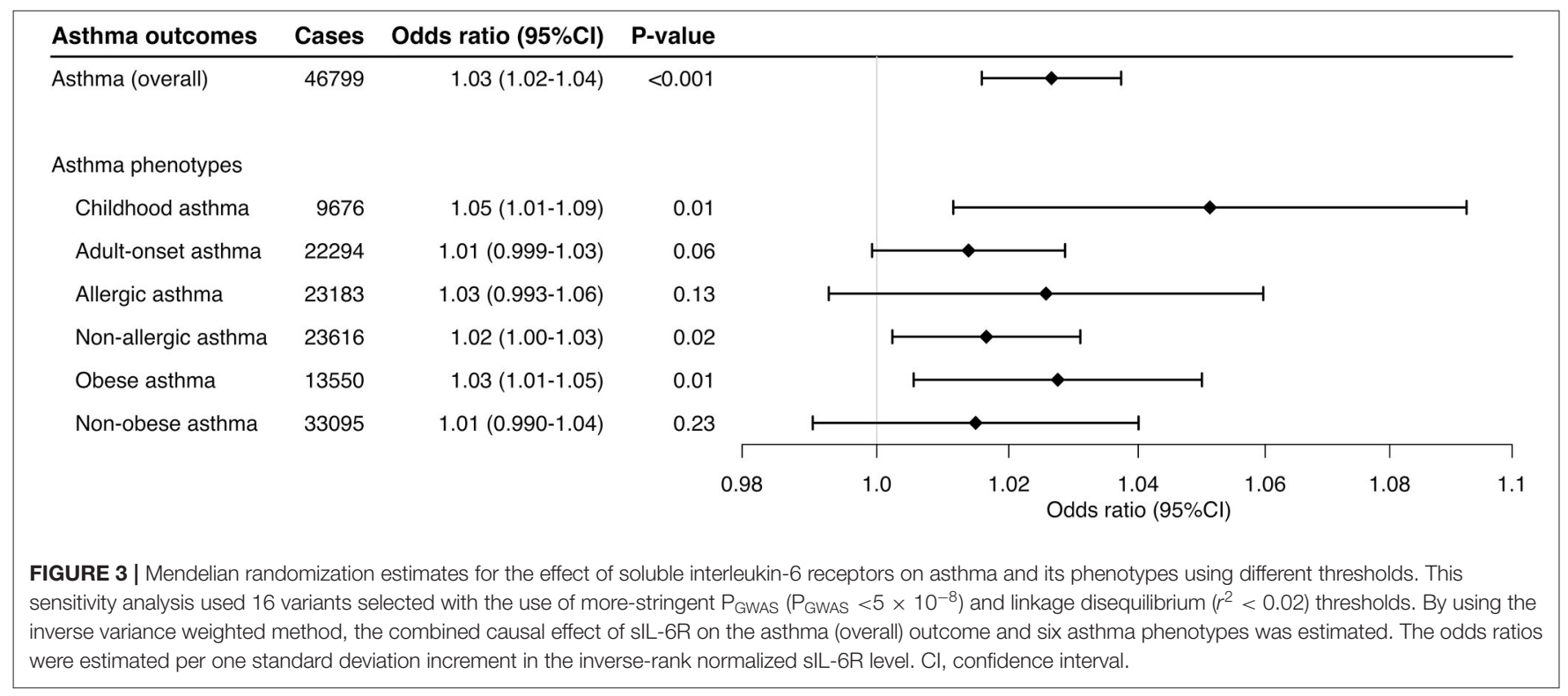

small sample size of patients with moderate-to-severe asthma precluded us from robustly examining this specific group that may benefit more from anti-IL-6 therapies. Third, as with any Mendelian randomization study, survivor (selection) bias is possible. However, participants from the INTERVAL study (mean age, 44 years) and UK Biobank (mean age, 57 years) were not in age ranges where survivor bias imposes a substantial impact. Fourth, the current study design 
using summary statistics precluded us from evaluating a potential non-linear relationship of sIL-6R with the asthma outcomes. Fourth, the influence of genetic instruments may be abated or buffered by feedback mechanisms or developmental processes. Yet, such mechanisms would have diminished the genetic effects, thereby biasing the inferences toward the null. Lastly, to minimize the population stratification bias, we restricted the study sample to individuals of European ancestry. Therefore, the inferences may not be generalizable to other racial/ethnic populations.

In conclusion, the current Mendelian randomization study using two large cohort data demonstrated that higher genetically-instrumented sIL-6R levels are associated with a significantly but modestly increased risk of overall asthma. The observation was consistent across the asthma phenotypes and different assumptions. Our inferences support further research into delineating the roles of IL-6 pathways in the asthma pathobiology and identifying patients with a distinct endotype who would benefit most from anti-IL-6 therapies.

\section{DATA AVAILABILITY STATEMENT}

The raw data supporting the conclusions of this article will be made available by the authors, without undue reservation.

\section{ETHICS STATEMENT}

The studies involving human participants were reviewed and approved by all participants from this study provided UK Biobank-acquired informed consent and provided data according to the UK Biobank protocol. The current study has complied with all ethical regulations according to UK Biobank policy. This research was approved and conducted using the UK Biobank under application number 16549 and 45052. The institutional review board of Harvard University and Massachusetts General Hospital approved the study. The patients/participants provided their written informed consent to participate in this study.

\section{REFERENCES}

1. Rose-John S. IL-6 Trans-signaling via the soluble IL-6 receptor: importance for the pro-inflammatory activities of IL-6. Int J Biol Sci. (2012) 8:123747. doi: 10.7150/ijbs.4989

2. Yokoyama A, Kohno N, Fujino S, Hamada H, Inoue Y, Fujioka S, et al. Circulating interleukin-6 levels in patients with bronchial asthma. Am J Respir Crit Care Med. (1995) 151:1354-8. doi: 10.1164/ajrccm.151.5.7735584

3. Neveu WA, Allard JL, Raymond DM, Bourassa LM, Burns SM, Bunn JY, et al. Elevation of IL-6 in the allergic asthmatic airway is independent of inflammation but associates with loss of central airway function. Respir Res. (2010) 11:28. doi: 10.1186/1465-9921-11-28

4. Zhang J-G, Chen X-J, Liu T, Jiang S-J. FOXP3+ associated with the proinflammatory regulatory $\mathrm{T}$ and $\mathrm{T}$ helper 17 effector cells in asthma patients. Exp Ther Med. (2016) 12:2753-8. doi: 10.3892/etm.2016.3662

5. Farahi N, Paige E, Balla J, Prudence E, Ferreira RC, Southwood M, et al. Neutrophil-mediated IL-6 receptor trans-signaling and the risk of chronic

\section{AUTHOR CONTRIBUTIONS}

YR carried out the main statistical analysis, drafted the initial manuscript, and approved the final manuscript as submitted. ZZ created the summary statistics of UK Biobank, drafted the initial manuscript, and approved the final manuscript as submitted. CC conceptualized and designed the study, supervised the conduct of study, critically reviewed and revised the initial manuscript, and approved the final manuscript as submitted. RF assisted study design, reviewed the manuscript, and approved the final manuscript. LL conceptualized the study, obtained funding and reviewed the manuscript, and approved the final manuscript. DN conceptualized the study, reviewed the manuscript, and approved the final manuscript. $\mathrm{KH}$ conceptualized the study, obtained funding, supervised the statistical analysis, reviewed and revised the initial manuscript, and approved the final manuscript as submitted. All authors contributed to the article and approved the submitted version.

\section{FUNDING}

This study was supported by grants (R01 AI-134940 and R01 AI148338) from the National Institutes of Health (Bethesda, MD). The content of this manuscript is solely the responsibility of the authors and does not necessarily represent the official views of the National Institutes of Health. The funding organization was not involved in the collection, management, or analysis of the data; preparation or approval of the manuscript; or decision to submit the manuscript for publication.

\section{ACKNOWLEDGMENTS}

This research was conducted using the UK Biobank Resource under Applications \#16549 and \#45052.

\section{SUPPLEMENTARY MATERIAL}

The Supplementary Material for this article can be found online at: https://www.frontiersin.org/articles/10.3389/fmed. 2021.665057/full\#supplementary-material

obstructive pulmonary disease and asthma. Hum Mol Genet. (2017) 26:158496. doi: 10.1093/hmg/ddx053

6. Hawkins GA, Robinson MB, Hastie AT, Li X, Li H, Moore WC, et al. The IL6R variation Asp(358)Ala is a potential modifier of lung function in subjects with asthma. J Allergy Clin Immunol. (2012) 130:5105.e1. doi: 10.1016/j.jaci.2012.03.018

7. Ferreira MAR, Matheson MC, Duffy DL, Marks GB, Hui J, Le Souëf $\mathrm{P}$, et al. Identification of IL6R and chromosome $11 \mathrm{q} 13.5$ as risk loci for asthma. Lancet. (2011) 378:1006-14. doi: 10.1016/S0140-6736(11) 60874-X

8. Sun BB, Maranville JC, Peters JE, Stacey D, Staley JR, Blackshaw J, et al. Genomic atlas of the human plasma proteome. Nature. (2018) 558:739. doi: 10.1038/s41586-018-0175-2

9. Sudlow C, Gallacher J, Allen N, Beral V, Burton P, Danesh J, et al. UK biobank: an open access resource for identifying the causes of a wide range of complex diseases of middle and old age. PLoS Med. (2015) 12:e1001779. doi: 10.1371/journal.pmed.1001779 
10. Zhu Z, Lee PH, Chaffin MD, Chung W, Loh P-R, Lu Q, et al. A genomewide cross-trait analysis from UK Biobank highlights the shared genetic architecture of asthma and allergic diseases. Nat Genet. (2018) 50:85764. doi: 10.1038/s41588-018-0121-0

11. Zhu Z, Guo Y, Shi H, Liu C-L, Panganiban RA, Chung W, et al. Shared genetic and experimental links between obesity-related traits and asthma subtypes in UK Biobank. J Allergy Clin Immunol. (2020) 145:53749. doi: 10.1016/j.jaci.2019.09.035

12. Zhu Z, Zhu X, Liu C-L, Shi H, Shen S, Yang Y, et al. Shared genetics of asthma and mental health disorders: a large-scale genome-wide cross-trait analysis. Eur Respir J. (2019) 54:1901507. doi: 10.1183/13993003.01507-2019

13. Davies NM, Holmes MV, Davey Smith G. Reading Mendelian randomisation studies: a guide, glossary, and checklist for clinicians. BMJ. (2018) 362:k601. doi: 10.1136/bmj.k601

14. Bowden J, Spiller W, Del Greco M F, Sheehan N, Thompson J, Minelli C, et al. Improving the visualization, interpretation and analysis of two-sample summary data Mendelian randomization via the Radial plot and Radial regression. Int J Epidemiol. (2018) 47:1264-78. doi: 10.1093/ije/dyy101

15. Burgess S, Dudbridge F, Thompson SG. Combining information on multiple instrumental variables in Mendelian randomization: comparison of allele score and summarized data methods. Stat Med. (2016) 35:1880906. doi: 10.1002/sim.6835

16. Staley OYJ. MendelianRandomization: Mendelian Randomization Package. (2020). Available online at: https://CRAN.R-project.org/package= MendelianRandomization (accessed November 2, 2020).

17. Burgess S, Thompson SG. Interpreting findings from Mendelian randomization using the MR-Egger method. Eur J Epidemiol. (2017) 32:377-89. doi: 10.1007/s10654-017-0255-x

18. Verbanck M, Chen C-Y, Neale B, Do R. Detection of widespread horizontal pleiotropy in causal relationships inferred from Mendelian randomization between complex traits and diseases. Nature Genetics. (2018) 50:6938. doi: 10.1038/s41588-018-0099-7

19. Bowden J, Davey Smith G, Haycock PC, Burgess S. Consistent estimation in Mendelian randomization with some invalid instruments using a weighted median estimator. Genet Epidemiol. (2016) 40:304-14. doi: 10.1002/gepi.21965

20. Jackson DJ, Bacharier LB, Calatroni A, Gill MA, Hu J, Liu AH, et al. Serum IL-6: a biomarker in childhood asthma? J Allergy Clin Immunol. (2020) 145:1701-4.e3. doi: 10.1016/j.jaci.2020.01.021

21. Peters MC, McGrath KW, Hawkins GA, Hastie AT, Levy BD, Israel E, et al. Plasma interleukin-6 concentrations, metabolic dysfunction, and asthma severity: a cross-sectional analysis of two cohorts. Lancet Respir Med. (2016) 4:574-84. doi: 10.1016/S2213-2600(16)3 0048-0

22. Li X, Hastie AT, Peters MC, Hawkins GA, Phipatanakul W, Li H, et al. Investigation of the relationship between IL- 6 and type 2 biomarkers in patients with severe asthma. J Allergy Clin Immunol. (2020) 145:4303. doi: 10.1016/j.jaci.2019.08.031
23. Zhu Z, Hasegawa K, Camargo CA, Liang L. Investigating asthma heterogeneity through shared and distinct genetics: insights from genome-wide cross-trait analysis. J Allergy Clin Immunol. (2020) 147:796-807. doi: 10.1016/j.jaci.2020.07.004

24. Franchimont N, Lambert C, Huynen P, Ribbens C, Relic B, Chariot A, et al. Interleukin- 6 receptor shedding is enhanced by interleukin-1beta and tumor necrosis factor alpha and is partially mediated by tumor necrosis factor alpha-converting enzyme in osteoblast-like cells. Arthritis Rheum. (2005) 52:84-93. doi: 10.1002/art.20727

25. Jevnikar Z, Östling J, Ax E, Calvén J, Thörn K, Israelsson E, et al. Epithelial IL-6 trans-signaling defines a new asthma phenotype with increased airway inflammation. J Allergy Clin Immunol. (2019) 143:57790. doi: 10.1016/j.jaci.2018.05.026

26. Jones SA, Novick D, Horiuchi S, Yamamoto N, Szalai AJ, Fuller GM. Creactive protein: a physiological activator of interleukin 6 receptor shedding. $J$ Exp Med. (1999) 189:599-604. doi: 10.1084/jem.189.3.599

27. Hurst SM, Wilkinson TS, McLoughlin RM, Jones S, Horiuchi S, Yamamoto N, et al. Il-6 and its soluble receptor orchestrate a temporal switch in the pattern of leukocyte recruitment seen during acute inflammation. Immunity. (2001) 14:705-14. doi: 10.1016/S1074-7613(01)00151-0

28. Walev I, Vollmer P, Palmer M, Bhakdi S, Rose-John S. Pore-forming toxins trigger shedding of receptors for interleukin 6 and lipopolysaccharide. Proc Natl Acad Sci USA. (1996) 93:7882-7. doi: 10.1073/pnas.93.15.7882

29. Yan I, Schwarz J, Lücke K, Schumacher N, Schumacher V, Schmidt S, et al. ADAM17 controls IL-6 signaling by cleavage of the murine IL-6R $\alpha$ from the cell surface of leukocytes during inflammatory responses. J Leukoc Biol. (2016) 99:749-60. doi: 10.1189/jlb.3A0515-207R

30. Peters U, Dixon AE, Forno E. Obesity and asthma. J Allergy Clin Immunol. (2018) 141:1169-79. doi: 10.1016/j.jaci.2018.02.004

31. Ullah MA, Revez JA, Loh Z, Simpson J, Zhang V, Bain L, et al. Allergeninduced IL- 6 trans-signaling activates $\gamma \delta \mathrm{T}$ cells to promote type 2 and type 17 airway inflammation. J Allergy Clin Immunol. (2015) 136:106573. doi: $10.1016 /$ j.jaci.2015.02.032

Conflict of Interest: CC has participated in scientific advisory boards for AstraZeneca and GSK. KH has received a research grant from Novartis.

The remaining authors declare that the research was conducted in the absence of any commercial or financial relationships that could be construed as a potential conflict of interest.

Copyright (c) 2021 Raita, Zhu, Camargo, Freishtat, Ngo, Liang and Hasegawa. This is an open-access article distributed under the terms of the Creative Commons Attribution License (CC BY). The use, distribution or reproduction in other forums is permitted, provided the original author(s) and the copyright owner(s) are credited and that the original publication in this journal is cited, in accordance with accepted academic practice. No use, distribution or reproduction is permitted which does not comply with these terms. 